\title{
Requerimientos pregerminativos de Maihuenia patagonica y Maihueniopsis darwinii, cactáceas endémicas de Patagonia
}

\section{Pre-germinative requirements of Maihuenia patagonica and Maihueniopsis darwinii, endemic cacti from Patagonia}

\author{
Ana Carla Ailín Masini ${ }^{1,2^{*}}$, Adriana Edit Rovere ${ }^{1,2}$ \& Gabriela Inés PIRK ${ }^{1,2,3}$ \\ ${ }^{1}$ Laboratorio Ecotono, Centro Regional Universitario Bariloche, Universidad Nacional del Comahue. Quintral 1250, San Carlos \\ de Bariloche (8400), Río Negro, Argentina. \\ ${ }^{2}$ Consejo Nacional de Investigaciones Científicas y Técnicas (CONICET). \\ ${ }^{3}$ Instituto de Investigaciones en Biodiversidad y Medioambiente (INIBIOMA, CONICET-UNCOMA). Quintral 1250, San Carlos \\ de Bariloche (8400), Río Negro, Argentina. \\ *carlitamasini@yahoo.com.ar
}

\begin{abstract}
RESUMEN
Las cactáceas son plantas características de ecosistemas áridos, en los cuales desarrollan un importante papel ecológico. Se encuentran amenazadas por la destrucción del hábitat y la extracción con fines comerciales. Argentina es uno de los países de Sudamérica con mayor riqueza y nivel de endemismo de cactáceas, sin embargo, su estudio es escaso, especialmente para las especies de distribución más austral. Dado que un mayor conocimiento sobre la biología reproductiva de los cactus ayudaría a mejorar su conservación, se estudiaron aspectos físiológicos básicos sobre la germinación de dos especies endémicas de Argentina y Chile, Maihuenia patagonica (Phil.) Britton \& Rose y Maihueniopsis darwinii (Hensl.) F. Ritter var. hickenii (Britton \& Rose) R. Kiesling. Se evaluó el porcentaje de germinación (G), el tiempo medio de germinación (TMG) y los días transcurridos hasta el inicio de la germinación (IG) de un control y de tres tratamientos pre-germinativos: estratificación húmeda fría, escarificación mecánica con lija y arena, y escarificación mecánica con bisturí. El ensayo se extendió por 60 días, en cámara de germinación a $20 / 10^{\circ} \mathrm{C}, 12 \mathrm{~h}$ luz/12 h oscuridad. Para M. patagonica la germinación no difirió entre la estratificación y el control, pero fue mayor al control en la escarificación con bisturí. Para M. darwinii var. hickenii la germinación sólo difirió entre la estratificación y la escarificación con bisturí. El TMG y el IG fueron mayores para la estratificación que para el control en M. patagonica, mientras que los tratamientos de escarificación no difirieron del control. En el caso de M. darwinii var. hickenii no se observaron diferencias en el TMG, pero el IG fue menor en la estratificación que en el control. Es posible que ambas especies presenten algún tipo de dormancia, ya que los porcentajes de germinación fueron bajos (M. patagonica de 8-27\% y M. darwinii var. hickenii de 17-26\%), pero las semillas presentaron elevada viabilidad. Se descarta la existencia de dormancia física debido a que hubo germinación en el control de ambas especies y se propone la existencia de dormancia fisiológica leve, la cual habría sido superada parcialmente por la escarificación mecánica con bisturí del tegumento en $M$. patagonica. Los resultados obtenidos constituyen un nuevo aporte al estudio de las cactáceas de Sudamérica, que puede ser utilizado para el desarrollo de medidas de conservación in situ mediante la restauración de hábitats naturales degradados.
\end{abstract}

Palabras clave: Dormancia, Maihuenioideae, Opuntioideae, Payunia, zonas áridas.

\begin{abstract}
Cacti are characteristic plants in arid ecosystems, where they play an important ecological role. Many species are threatened because of habitat destruction and collection for commercial purposes. In South America, Argentina is one of the countries with the greatest cacti richness and endemisms. However, cacti have been scarcely studied, especially the southernmost species. Since the study of cacti's reproductive biology would help to improve their conservation status, we studied basic physiological aspects of the germination of two endemic species of Argentina and Chile, Maihuenia patagonica (Phil.) Britton \& Rose and Maihueniopsis darwinii (Hensl.) F. Ritter var. hickenii (Britton \& Rose) R. Kiesling. Germination percentage $(\mathrm{G})$, mean germination time (TMG) and time until germination (IG) of control seeds and seeds subjected to three pre-germinative treatments were evaluated. Treatments were cold moist stratification for 40 days, mechanical scarification with sand and sandpaper, and mechanical scarification with a scalpel. Laboratory assays were performed at 20/10 ${ }^{\circ} \mathrm{C}$ and $12 \mathrm{~h} \mathrm{light} / 12 \mathrm{~h}$ dark regime during 60 days. In M. patagonica germination in the stratification treatment did not differ from the control, but was higher in the scarification with scalpel. In M. darwinii var. hickenii germination only
\end{abstract}


differed between the stratification and the scarification with scalpel. TMG and IG were higher in the stratification treatment than in the control for M. patagonica, whereas scarification treatments did not differ from the control. As for M. darwinii var. hickenii no differences were detected for TMG, but the IG was lower in the stratification than in the control. Probably both species presents some kind of dormancy, since germination percentages were low (M. patagonica between 8-27\% and M. darwinii var. hickenii between 17-26\%) but seeds presented high viability. Presence of physical dormancy is discarded because control seeds of both species germinated and the presence of non deep physiological dormancy is proposed. The latter was partially overcome by mechanical scarification in $M$. patagonica. These results are a new contribution to the study of cacti in South America and could help to develop in situ conservation strategies through the restoration of degraded areas.

KEYwords: Arid zones, dormancy, Maihuenioideae, Opuntioideae, Payunia.

\section{INTRODUCCIÓN}

La familia Cactaceae constituye un taxón de interés tanto por su distribución como por sus características biológicas y su estado de conservación. Las cactáceas son endémicas de América (Anderson 2001), siendo una familia de plantas característica de ecosistemas áridos, donde desempeñan un importante papel ecológico (Ortega-Baes \& GodínezÁlvarez 2006). Presentan adaptaciones morfofisiológicas a la baja disponibilidad de agua y una gran diversidad de formas de crecimiento (Anderson 2001). Debido a que poseen ciclos de vida prolongados, bajas tasas de crecimiento, estadios tempranos vulnerables y distribución generalmente restringida, son sensibles a disturbios ambientales (Godínez-Álvarez et al. 2003, Ortega-Baes \& Godínez-Álvarez 2006). Por ello se encuentran amenazadas o en peligro de extinción principalmente por la destrucción del hábitat y la extracción con fines comerciales (Oldfield 1997, CITES 2013). De esta manera, es fundamental estudiarlas para contribuir a su conservación.

Entre las posibles estrategias de conservación y reintroducción de plantas, son particularmente importantes aquéllas enfocadas en preservar la diversidad genética de las especies. Si bien las cactáceas en general pueden reproducirse vegetativamente (Anderson 2001), la propagación a partir de semillas permite preservar su diversidad genética (RojasAréchiga \& Vázquez-Yanes 2000). El almacenamiento en bancos de semillas (Ortega-Baes et al. 2010c) constituye una reserva a largo plazo de la diversidad genética y una fuente de material para trabajos de restauración (Guerrant \& Raven 2003). La recuperación tanto de poblaciones naturales como de hábitats degradados (ver por ejemplo Stiling et al. 2000, Bacilio et al. 2006, Woods et al. 2012) constituyen alternativas que permitirían mejorar su estado de conservación. Por lo tanto, para llevar a cabo trabajos de restauración deben conocerse aspectos de la biología reproductiva de las especies de interés, por ejemplo, las características de sus semillas y sus requerimientos de germinación, a fin de reintroducirlas en su ambiente natural (Martínez Pérez et al. 2006, Commander et al. 2009, Masini et al. 2012).
Las semillas de las cactáceas suelen ser ortodoxas y permanecer viables durante un tiempo variable al ser almacenadas (Rojas-Aréchiga \& Vázquez-Yanes 2000) o en los bancos de semillas del suelo (Godínez-Álvarez et al. 2003). La temperatura óptima de germinación es de $20^{\circ} \mathrm{C}$, viéndose desfavorecida por debajo de $12{ }^{\circ} \mathrm{C}$ y por encima de $28{ }^{\circ} \mathrm{C}$ (Rojas-Aréchiga \& Vázquez-Yanes 2000). Con relación al requerimiento lumínico, se ha observado que las semillas pequeñas generalmente son fotoblásticas positivas, y que el fotoblastismo es independiente de la presencia de mecanismos de dormancia, del hábito y se relaciona en cierta medida con la filogenia de las especies (Flores et al. 2011, Rojas-Aréchiga et al. 2013). Según la revisión realizada por Rojas-Aréchiga \& Vázquez-Yanes (2000), se han observado mecanismos de dormancia innata e impuesta en distintas especies de cactus, y se ha atribuido la ausencia o baja germinación a diversas causas (compuestos inhibidores en el tegumento de la semilla, embriones inmaduros, cubiertas impermeables que necesitan pasar por el tracto digestivo de un animal o escarificación, requerimiento de estratificación). Teniendo en cuenta un sistema de clasificación de dormancia desarrollado más recientemente, los mecanismos observados corresponderían a dormancia fisiológica, morfológica y física, y a la existencia de semillas quiescentes (Baskin \& Baskin 2004). Cabe destacar que la respuesta germinativa depende en parte de la edad de las semillas (ver por ejemplo Kwack \& Zimmer 1978, Flores et al. 2005, Delgado-Sánchez et al. 2011). Sin embargo, la información disponible en cuanto a las características básicas de la germinación no es equivalente entre distintas áreas geográficas y géneros de cactáceas.

A pesar de la diversidad y el nivel de endemismo existentes en Sudamérica, el estudio de las cactáceas es reducido en comparación con México y otros países con diversidad similar (Ortega-Baes et al. 2010c). En particular, Argentina presenta unas 235 especies de cactus (Zuloaga et al. 2008), siendo uno de los países con mayor riqueza, y una proporción de especies endémicas del 61\% (OrtegaBaes \& Godínez-Alvarez 2006). Estudios sobre la biología reproductiva, y particularmente la germinación, de las especies nativas se ha desarrollado principalmente en 
poblaciones de cactáceas del norte del país (ver por ejemplo Cazón et al. 2002, Ortega-Baes \& Rojas-Aréchiga 2007, Ortega-Baes et al. 2010a, 2010b), y en menor medida en poblaciones del centro (ver por ejemplo Gurvich et al. 2008, Méndez \& Pérez González 2008, Méndez 2007, 2011) y sur (Kwack \& Zimmer 1978, Leuenberger 1997). Entre los géneros de distribución más austral se incluyen Maihuenia y Maihueniopsis.

Las especies Maihuenia patagonica (Phil.) Britton \& Rose (Subfamilia Maihuenioideae) y Maihueniopsis darwinii (Hensl.) F. Ritter (Subfamilia Opuntioideae) se distribuyen parcialmente en zonas áridas de la Patagonia (Zuloaga et al. 2008), donde las precipitaciones se concentran en invierno (Paruelo et al. 1998). Especies nativas de desiertos y semidesiertos con inviernos húmedos suelen presentar dormancia fisiológica, aunque también se observan otros tipos de dormancia específicos de cada familia (por ejemplo dormancia física en Fabaceae) (Baskin \& Baskin 1998). Considerando los mecanismos de dormancia observados en otras cactáceas (Rojas-Aréchiga \& Vázquez-Yanes 2000), el tipo de ambiente y la época de fructificación (verano-otoño), es posible que $M$. patagonica y $M$. darwinii presenten dormancia fisiológica o física. De observarse dormancia física, resultaría útil para trabajos de restauración evaluar técnicas de escarificación aplicables fácilmente a una gran cantidad de semillas en simultáneo, como es el caso de la abrasión mecánica (Bainbridge 2007). Asimismo, resulta de interés determinar el número de semillas por fruto, ya que dicha información es necesaria al momento de planear la colecta de semillas a campo (Way 2003), y el tamaño y peso de las semillas, información útil al momento de almacenarlas en bancos de semillas (Terry et al. 2003).

Dada la importancia de conocer las características de las semillas y de la germinación de las cactáceas para su almacenamiento en bancos de semillas y su utilización en proyectos de restauración, el presente trabajo indaga sobre aspectos fisiológicos básicos de la germinación de Maihuenia patagonica y Maihueniopsis darwinii var. hickenii (Britton \& Rose) R. Kiesling. Se plantean como objetivos: caracterizar el número de semillas por fruto, el tamaño y peso de las semillas, y evaluar la presencia de dormancia física y fisiológica.

\section{MATERIALES Y MÉTODOS}

\section{ESPECIES ESTUDIADAS}

El género Maihuenia es el único incluido en la subfamilia Maihuenioideae, que ocupa una posición basal dentro de la filogenia de las cactáceas, aunque su ubicación con respecto a las demás subfamilias aún se encuentra en discusión (ver por ejemplo Butterworth \& Wallace 2005, Las Peñas et al. 2014). Está constituido por las especies M. patagonica y $M$. poeppigii (Otto ex Pfeiff.) F.A.C. Weber, ambas distribuidas exclusivamente en Argentina y Chile (Anderson 2001), siendo después de Austrocactus el género de cactus de distribución más austral (Leuenberger 1997). Maihuenia patagonica se distribuye en Argentina en el sur de Mendoza, Neuquén, Río Negro, Chubut y Santa Cruz, y en Chile en las regiones X, XI y XII, entre 0-500 m s.n.m. (Zuloaga et al. 2008), aunque se han registrado ejemplares hasta 2.600 m s.n.m. (Leuenberger 1997). En Argentina, M. patagonica se distribuye principalmente en la provincia fitogeográfica Patagónica, y en menor medida en las provincias del Monte y Altoandina, y es considerada un elemento típico del semidesierto patagónico (Leuenberger 1997). Habita en una gran variedad de sustratos, particularmente de origen volcánico (Leuenberger 1997). Dentro de su rango de distribución la temperatura media anual es de $8-14{ }^{\circ} \mathrm{C}$ y la precipitación de 130-250 mm anuales, el período de sequía se extiende durante unos 8-10 meses, siendo esta especie resistente tanto a altas temperaturas del sustrato durante el verano como a temperaturas bajo cero durante el invierno, y presentando adaptaciones de resistencia a la sequía (Leuenberger 1997, Mauseth 1999).

Maihuenia patagonica (Fig. 1) (Kiesling 1975, 1988, Leuenberger 1997) forma arbustos en cojín, presenta aréolas con tres espinas aplanadas, una central de mayor longitud y dos más pequeñas laterales, y hojas cilíndricas, suculentas y persistentes. Las flores son acampanadas, con piezas petaloides blancas o rosadas. Los frutos son globosos, verdeamarillentos, con el perianto seco persistente. Tanto la pared del fruto como los funículos son mucilaginosos, sin embargo en frutos completamente maduros estas estructuras están secas. Las semillas son lenticulares, brillantes, negras, de 3 $\mathrm{mm}$ de diámetro y $2 \mathrm{~mm}$ de espesor, con un embrión curvo en forma de $\mathrm{U}$, que rodea un abundante perisperma harinosocalcáreo. La floración ocurre entre noviembre y enero, y la fructificación entre diciembre y marzo. Aparentemente, $M$. patagonica es autoincompatible al igual que $M$. poeppigii, y no se reproduciría asexualmente. En cuanto a su estado de conservación, $M$. patagonica no ha sido incluida en la base de datos de Plantas Endémicas de Argentina (PlanEAr) ya que presenta una extensa distribución en Chile (Testoni com. pers.), y es clasificada por la UICN dentro de la categoría de Preocupación Menor (Kiesling et al. 2013).

Maihueniopsis darwinii (subfamilia Opuntioideae) se distribuye exclusivamente en Argentina (Zuloaga et al. 2008). Maihueniopsis darwinii var. hickenii (Fig. 2) se distribuye en Buenos Aires, Mendoza, Neuquén, Río Negro, Chubut y Santa Cruz, entre 0-1.000 m s.n.m. (Zuloaga et al. 2008). Pertenece al género de cactáceas que llega a mayor altitud, tratándose de plantas adaptadas al clima frío, con heladas durante la noche, y a una intensa radiación solar durante el día (Kiesling 1984). Esta variedad (Kiesling 1984, 1988) presenta arbustos en forma de cojín de más de $1 \mathrm{~m}$ de diámetro, con artejos de hasta $3 \mathrm{~cm}$ de diámetro, color verde 
oliva, aréolas con pelos, gloquidios inconspicuos y espinas de hasta $12 \mathrm{~cm}$ de longitud, con un grueso nervio central y dos alas laterales, y hojas rápidamente caedizas. Las flores son acampanadas, con perigonio corto de piezas amarillas. Los frutos son carnosos, similares a los artejos, color amarilloanaranjado cuando muy maduros. Las semillas son globosas, lenticulares, de 5-6 $\mathrm{mm}$ de diámetro y $5 \mathrm{~mm}$ de espesor, con tegumento externo castaño, delgado y débil, y tegumento interno delgado y blancuzco, y un embrión curvo en forma de U rodeando un perisperma abundante. Las semillas están cubiertas por un arilo compacto, blando, color amarillocastaño. Con respecto a su reproducción, se ha observado que $M$. darwinii no se reproduce asexualmente o solo raramente (Kiesling 1984), sin embargo existe evidencia de que la especie se propagaría comúnmente de forma clonal (Méndez et al. 2004), lo cual es una característica típica de la subfamilia Opuntioideae (Anderson 2001). La propagación a través de semillas de esta especie se desconoce. En cuanto a su estado de conservación, $M$. darwinii recibe la categoría 3 de PlanEAr, es decir, se trata de una especie común, aunque no abundante en una o más de las unidades fitogeográficas del país (PlanEAr 2008), y la categoría de Preocupación Menor de la UICN (Demaio et al. 2013).

Se confeccionaron ejemplares de herbario de cada especie para su identificación taxonómica, que se encuentran depositados en el Herbario de la Universidad Nacional del Comahue (BCRU).
Sitio DE ESTUdio

El material fue recolectado en la Reserva Provincial de Usos Múltiples Auca Mahuida, ubicada al noreste de la provincia del Neuquén (Argentina), en el Distrito fitogeográfico de la Payunia. Este distrito es considerado un ecotono entre las Provincias del Monte y Patagónica (Cabrera 1971) y presenta un importante número de endemismos de plantas vasculares, razón por la cual se le adjudica un elevado interés biogeográfico (Mártinez Carretero 2004). El clima del sitio de estudio es semiárido (Mártinez Carretero 2004), y la precipitación media anual de la región es menor a 130 mm (Departamento de Geografía UNCo 1982). Durante el año 2012 se registró una precipitación anual de $185 \mathrm{~mm}$, concentrada principalmente entre enero-febrero y octubrenoviembre, una temperatura media anual de $10,5^{\circ} \mathrm{C}$, siendo la máxima temperatura estival de $31^{\circ} \mathrm{C}$ (enero) y la mínima invernal de $-9^{\circ} \mathrm{C}$ (junio) (datos registrados por una estación meteorológica local, ubicada a $37^{\circ} 42^{\prime} \mathrm{S}, 68^{\circ} 51^{\prime} \mathrm{O}, 1.549 \mathrm{~m}$ s.n.m.). En la reserva se realizan actividades de exploración y explotación de hidrocarburos, entre cuyos impactos se encuentran canteras de extracción de áridos, caminos denudados de vegetación y explanadas petroleras (Fiori \& Zalba 2003). Cabe destacar que tanto $M$. patagonica como M. darwinii var. hickenii (en adelante M. darwinii) sólo fueron observadas en áreas no alteradas dentro de la reserva.

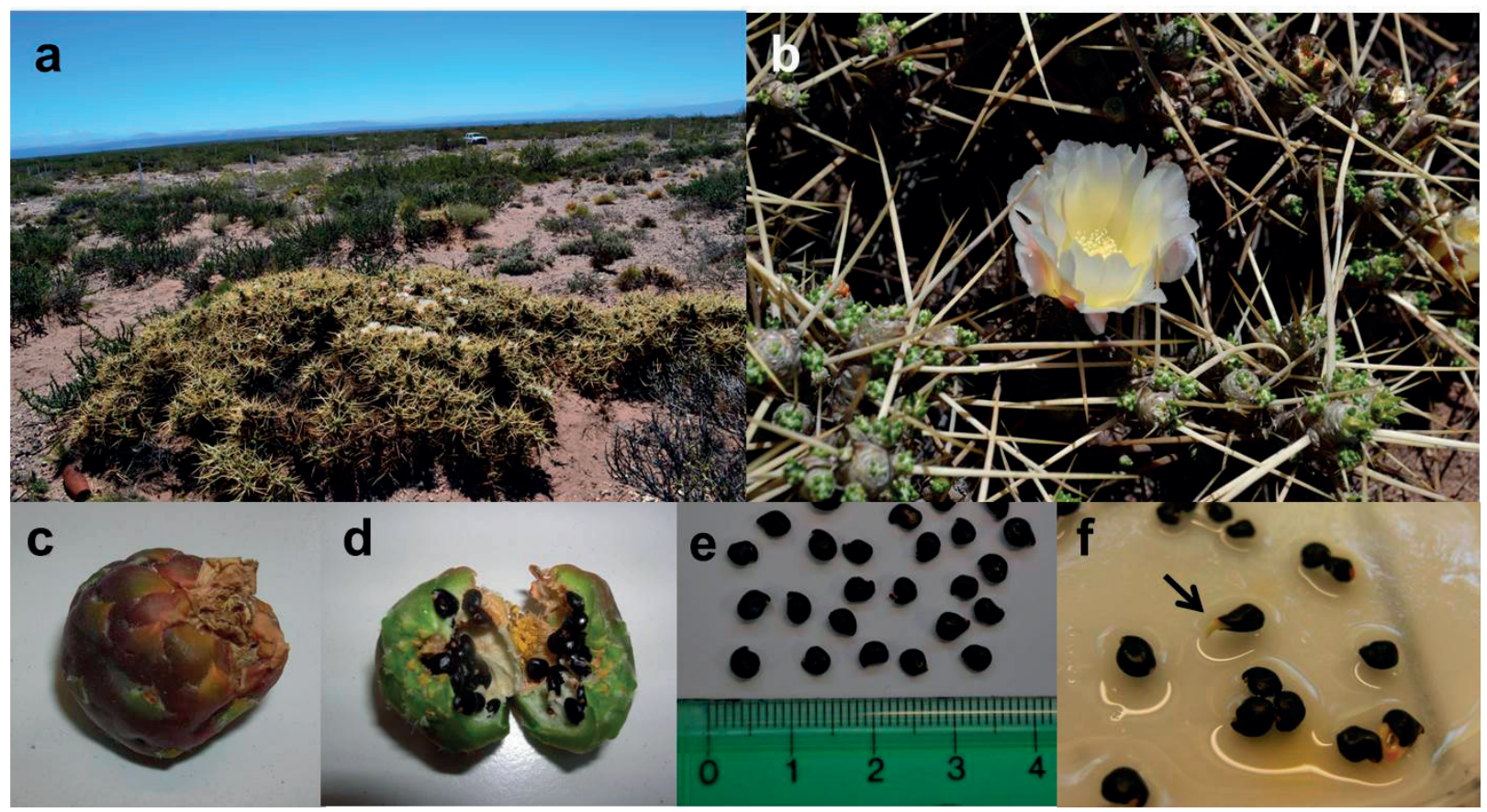

Figura 1. Maihuenia patagonica: a) ejemplar durante la floración, b) detalle de flor y tallo, con las hojas cilíndricas carnosas que caracterizan al género, c) fruto, d) fruto seccionado longitudinalmente con semillas en su interior, e) semillas junto a una escala en cm, f) semilla germinada durante el ensayo de germinación.

FiguRE 1. Maihuenia patagonica: a) individual during flowering, b) flower and stem with fleshy cylindrical leaves which characterize the genus, c) fruit, d) longitudinally dissected fruit with seeds inside, e) seeds with cm scale, f) germinated seed during germination assay. 


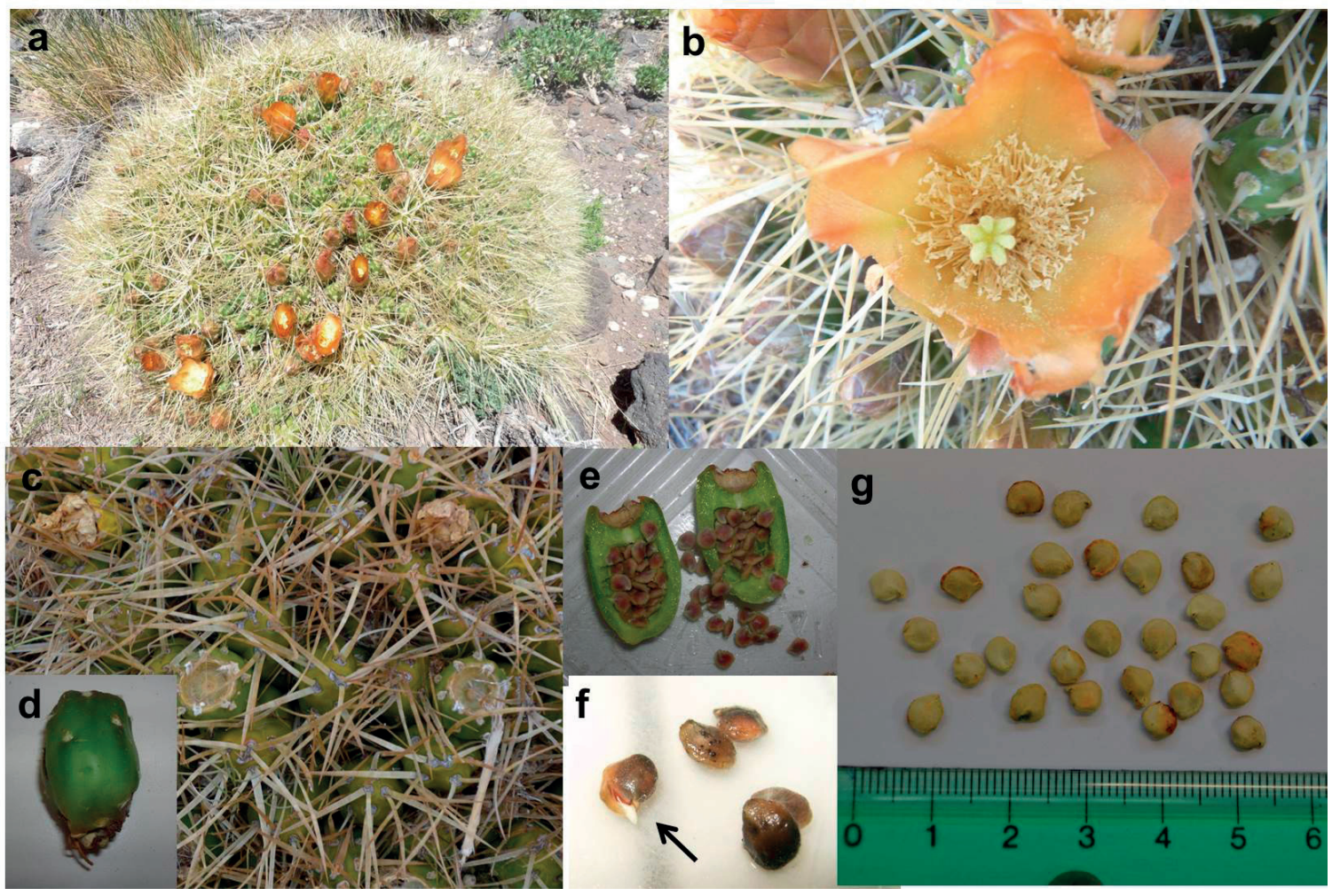

Figura 2. Maihueniopsis darwinii var. hickenii: a) ejemplar durante la floración, b) detalle de la flor, c) ejemplar con frutos, d) fruto, e) fruto seccionado longitudinalmente con semillas en su interior, f) semilla germinada durante el ensayo de germinación, g) semillas junto a una escala en $\mathrm{cm}$.

Figure 2. Maihueniopsis darwinii var. hickenii: a) individual during flowering, b) flower, c) individual with fruits, d) fruit, e) longitudinally dissected fruit with seeds inside, e) germinated seed during germination assay, f) seeds with $\mathrm{cm}$ scale.

\section{RECOLECCIÓN DE MATERIAL Y ENSAYO DE LABORATORIO}

Se recolectaron frutos maduros de 20 plantas de cada especie en febrero de 2013 (37 $41^{\circ}$ 'S; 68 50 ' O; 1.441 $\mathrm{m}$ s.n.m.). En laboratorio se extrajeron las semillas de los frutos, se enjuagaron bajo chorro de agua corriente para eliminar espinas y gloquidios, y se dejaron secar a temperatura ambiente. Todas las semillas de una misma especie se juntaron en un único lote, se guardaron en bolsa de papel rotulada, y se almacenaron en una heladera a $5^{\circ} \mathrm{C}$, en oscuridad. Las semillas fueron almacenadas durante 60 días previos a la realización del ensayo de germinación.

Se registró el número de semillas por fruto, considerando solamente frutos completos. Se midieron las dimensiones de 100 semillas de cada especie, utilizando un calibre digital. A cada semilla se le midió la longitud máxima, el ancho máximo y el espesor (Sánchez et al. 2002). Se pesaron diez lotes de 100 semillas de cada especie utilizando una balanza de precisión (Metter AJ150).

Se realizó un ensayo de germinación aplicando tres tratamientos pregerminativos y un control (C): 1) estratificación húmeda fría durante 40 días (EHF), en oscuridad; 2) escarificación mecánica con arena y lija (EML), sacudiéndose durante 2 min las semillas dentro de un recipiente revestido con lija de grano grueso y con 1/3 de su volumen de arena; y 3) escarificación mecánica con bisturí (EMB), retirándose con bisturí una porción del tegumento de la semilla en el caso de $M$. patagonica, y una porción del arilo hasta que quedara expuesto el tegumento de la semilla en el caso de $M$. darwinii. Para cada tratamiento se realizaron diez repeticiones de 30 semillas cada una. Las semillas se desinfectaron con una solución de hipoclorito de sodio al 2\% durante 2 min, se enjuagaron durante 2 min bajo chorro de agua corriente y se colocaron en cajas de Petri de plástico, sobre un disco de papel de filtro humedecido con agua destilada. Se llevaron a una cámara de germinación bajo condiciones controladas de luz y temperatura durante 64 días: 12 h luz / 12 h oscuridad, a 20/10 ${ }^{\circ} \mathrm{C}$, respectivamente. Se controló la germinación día por medio, regándose cuando fuera necesario con agua destilada. Se consideró germinada una semilla cuando emergía la radícula.

Una vez finalizado el ensayo de germinación, se realizó la prueba de viabilidad de tetrazolio a las semillas que no germinaron. Para esto se preacondicionaron las semillas, escarificándolas con bisturí hasta que fuera visible el embrión y colocándolas en un recipiente con agua destilada, en una incubadora a $30^{\circ} \mathrm{C}$, en oscuridad, durante 
24 h. Luego se las colocó en una solución de cloruro de 2,3,5-trifeniltetrazolio al 1\%, preparada con agua destilada, y se las volvió a llevar a la incubadora a $30^{\circ} \mathrm{C}$, en oscuridad durante $72 \mathrm{~h}$. Las semillas se cortaron longitudinalmente con un bisturí y se observaron bajo lupa estereoscópica, y se clasificaron en vacías, atacadas por hongos, infestadas (atacadas por insectos), viables y no viables.

El porcentaje de germinación final $(G)$ para cada repetición se calculó utilizando la fórmula $\mathrm{G}=\mathrm{g}$ / $(\mathrm{g}+\mathrm{tzp}+\mathrm{tzn}+\mathrm{h})$, donde $\mathrm{g}$ es el número de semillas germinadas, tzp es el número de semillas viables según la prueba de tetrazolio, tzn es el número de semillas no viables según la prueba de tetrazolio y h es el número de semillas atacadas por hongos (Gosling 2003). Del cálculo del porcentaje de germinación se excluyeron las semillas vacías e infestadas, es decir, aquellas que no tenían la potencialidad de germinar (Gosling 2003). Además se calculó el tiempo medio de germinación (TMG) (Hartmann \& Kester 1980, Yang et al. 2003) y los días transcurridos hasta el inicio de la germinación (IG) (Méndez 2007).

Para comparar los porcentajes finales de germinación entre tratamientos, y el TMG e IG en $M$. darwinii, se aplicó un test de ANOVA de una vía, y en caso de que fuera significativo se aplicaron comparaciones de medias a posteriori utilizando el test de Bonferroni. Para la comparación del TMG e IG en $M$. patagonica se aplicó el test no paramétrico de Kruskal-Wallis, y en caso de que fuera significativo se aplicaron comparaciones múltiples a posteriori de los rangos promedio (Siegel \& Castellan 1995). Para poder calcular el TMG debió eliminarse del análisis una de las repeticiones del tratamiento EML en la cual no se observó germinación para $M$. patagonica. El análisis de los datos se realizó trabajando con un $\alpha$ del 5\%. Se graficaron además las curvas de germinación acumulada en función del tiempo para cada tratamiento.

\section{RESULTADOS}

En la Tabla I se indica el número de semillas por fruto, y los resultados obtenidos con respecto a las dimensiones y peso de las semillas para ambas especies. El rango de variación del número de semillas por fruto para $M$. patagonica fue de 7-141 semillas/fruto y para $M$. darwinii de 52-111 semillas/ fruto.

El rango de germinación observado para $M$. patagonica fue de $8-27 \%$ y para $M$. darwinii de $17-26 \%$, considerando el control y todos los tratamientos (Fig. 3). El porcentaje de germinación final difirió entre tratamientos para ambas especies (ANOVA: $M$. patagonica, $F_{(3,36)}=17,8, \mathrm{p}<0,001 ; M$. darwinii, $\left.F_{(3,36)}=3,3, \mathrm{p}<0,05\right)$. En el caso de $M$. patagonica, C, EHF y EML no difieren entre sí y difieren de EMB, que presenta un porcentaje de germinación mayor (Fig. 3a); mientras que para $M$. darwinii, los únicos tratamientos que difieren son EHF y EMB, ya que ninguno de ellos difiere de C y EML (Fig. 3b).

El desarrollo de la germinación se analizó mediante curvas de germinación acumulada en función del tiempo (Fig. 4). En el caso de M. patagonica (Fig. 4a) se observó que el porcentaje de germinación se estabilizó para $\mathrm{C}, \mathrm{EML}$ y EMB hacia el final del ensayo, mientras que para EHF la germinación continuó aumentando. Si bien la germinación del tratamiento EMB se desarrolló principalmente durante los primeros 20 días del ensayo, los demás tratamientos presentaron tasas de germinación menos pronunciadas. Para $M$. darwinii (Fig. 4b) se observó un desarrollo de la germinación similar en todos los tratamientos, con un aumento pronunciado de la tasa de germinación hasta los 30 días del ensayo y luego una disminución de la misma, siendo el porcentaje final alcanzado por EHF menor al de los demás tratamientos.

Con respecto al TMG y al IG, para $M$. patagonica se observaron diferencias entre los tratamientos para ambas variables (Kruskal-Wallis: TMG, $\mathrm{H}=12,2, \mathrm{p}<0,01$; $\mathrm{IG}$, $\mathrm{H}=19,3, \mathrm{p}<0,001)$, mientras que para $M$. darwinii el test de ANOVA resultó no significativo para el TMG $\left(F_{(3,36)}=2,0\right.$, $\mathrm{p}=0,13)$ y significativo para el IG $\left(F_{(3,36)}=15,4, \mathrm{p}<0,001\right)$ (Tabla II). Para $M$. patagonica, el C y EMB presentaron el menor TMG e IG, mientras que EHF presentó los mayores valores para ambas variables, y EML no difirió de los demás tratamientos ni del control. En el caso de $M$. darwinii el TMG promedio para el control y los tratamientos fue de

TABla I. Número de semillas por fruto (media $\pm 1 \mathrm{DE})$ de $M$. patagonica $(\mathrm{N}=43)$ y $M$. darwinii var. hickenii $(\mathrm{N}=21)$, dimensiones de una semilla $(\mathrm{N}=100)$ y peso de un lote de 100 semillas $(\mathrm{N}=10)$.

TABLE I. Number of seeds per fruit (mean $\pm 1 \mathrm{SD})$ of M. patagonica $(\mathrm{N}=43)$ and $M$. darwinii var. hickenii $(\mathrm{N}=21)$, seed dimensions $(\mathrm{N}=100)$ and weight of 100 seeds $(\mathrm{N}=10)$.

\begin{tabular}{l|c|c}
\hline & Maihuenia patagonica & Maihueniopsis darwinii var. hickenii \\
\hline Semillas/fruto & $52,60 \pm 34,50$ & $85,30 \pm 21,20$ \\
Largo (mm) & $3,90 \pm 0,30$ & $5,20 \pm 0,40$ \\
Ancho (mm) & $3,12 \pm 0,25$ & $4,41 \pm 0,35$ \\
Espesor (mm) & $1,86 \pm 0,19$ & $2,10 \pm 0,19$ \\
Peso $(\mathrm{g})$ & $1,08 \pm 0,03$ & $1,88 \pm 0,04$ \\
\hline
\end{tabular}


30,8 $\pm 6,3$ días (media $\pm 1 \mathrm{DE}$ ), y en cuanto al IG se observó que las semillas de EHF fueron las que más rápidamente comenzaron a germinar, seguidas por las de EMB y EML, mientras que no se observaron diferencias entre $\mathrm{C}$ y estos dos últimos tratamientos.

Al realizar la prueba de viabilidad de tetrazolio, se observó que en $M$. patagonica el porcentaje de semillas no germinadas que resultaron viables varió entre $0-73,3 \%$, siendo en promedio de $39,2 \pm 21,7 \%$, mientras que las
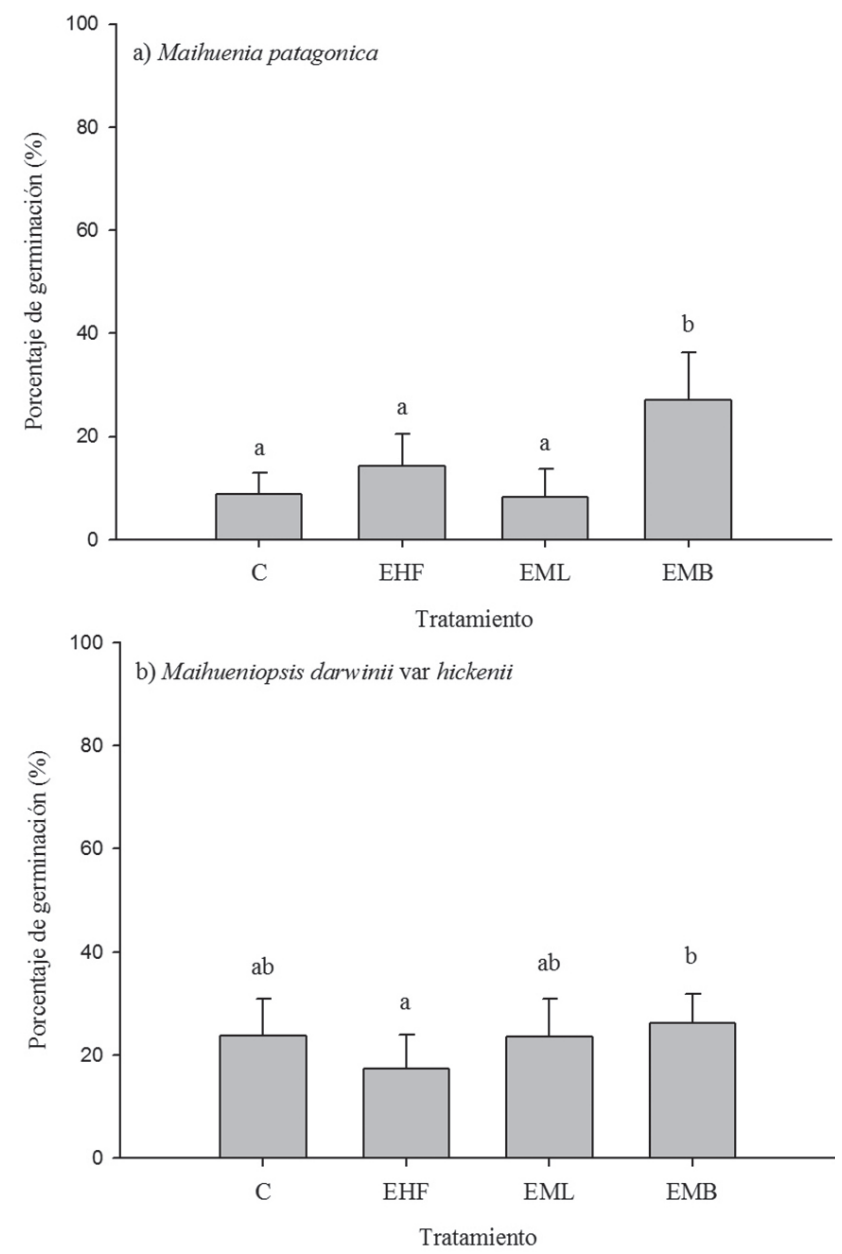

FIGURA 3. Porcentaje de germinación (media $\pm 1 \mathrm{DE})$ en semillas de a) Maihuenia patagonica y b) Maihueniopsis darwinii var. hickenii, en el control (C) y luego de la aplicación de tres tratamientos pregerminativos: estratificación húmeda fría durante 40 días (EHF), escarificación mecánica con lija y arena (EML), y escarificación mecánica con bisturí (EMB). Letras diferentes indican diferencias estadísticamente significativas entre tratamientos $(\mathrm{p}<0,05)$.

FIGURE 3. Germination percentage (mean $\pm 1 \mathrm{SD}$ ) in control seeds (C) of a) Maihuenia patagonica and b) Maihueniopsis darwinii var. hickenii, and seeds subjected to three pre-germinative treatments: cold moist stratification for 40 days (EHF), mechanical scarification with sand and sandpaper (EML), and mechanical scarification with a scalpel (EMB). Different letters indicate statistically significant differences among treatments $(\mathrm{p}<0.05)$. semillas no viables representaron entre $13,3-66,7 \%$, siendo en promedio de 42,2 $\pm 14,6 \%$. En el caso de $M$. darwinii los valores obtenidos fueron para semillas viables de entre $23,3-66,7 \%$, con una media de $44,0 \pm 10,0 \%$, y para semillas no viables entre $0-36,7 \%$, con una media de $19,8 \pm 8,9 \%$. Sin embargo, debe destacarse que las semillas que no se tiñeron o solo levemente, y que se consideraron no viables según esta técnica, presentaron embriones con aspecto sano (turgentes), que en el caso de haberse evaluado según un test de corte hubieran sido considerados viables (Gosling 2003). Las únicas semillas que con seguridad no se encontraban viables fueron aquéllas incluidas en las categorías de vacías, infestadas y atacadas por hongos.

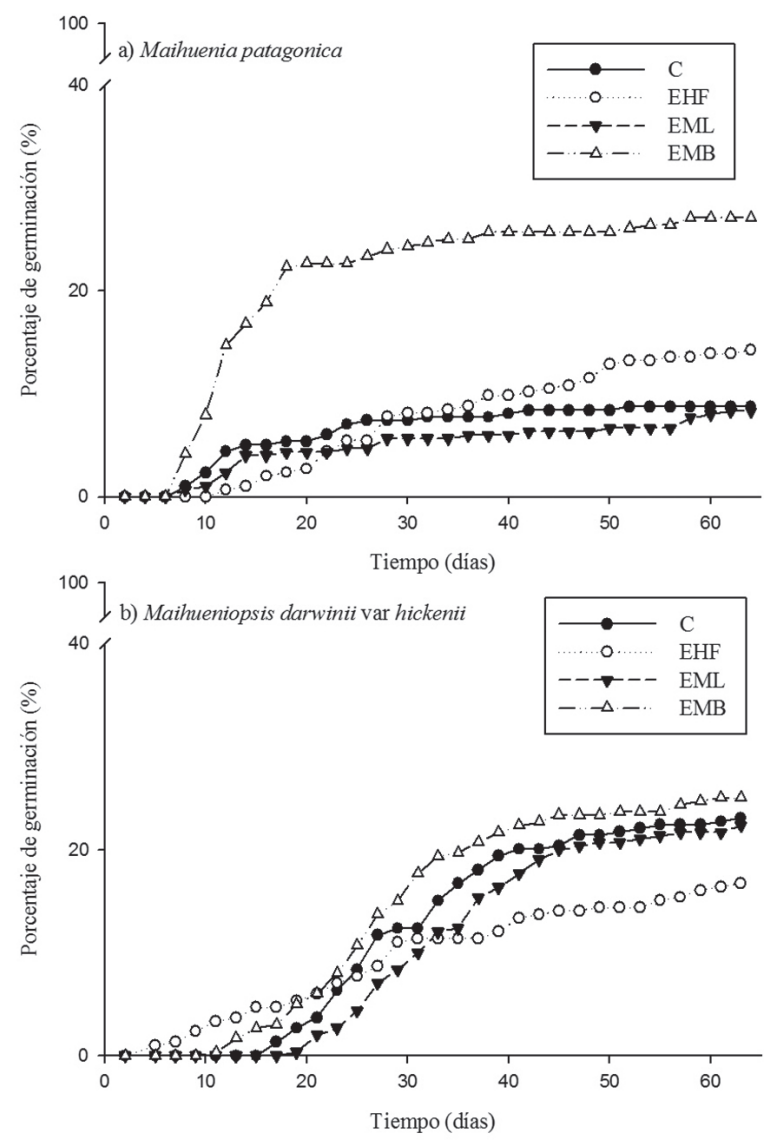

Figura 4. Curvas de germinación acumulada de a) Maihuenia patagonica y b) Maihueniopsis darwinii var. hickenii, en el control (C) y luego de la aplicación de tres tratamientos pregerminativos: estratificación húmeda fría durante 40 días (EHF), escarificación mecánica con lija y arena (EML), y escarificación mecánica con bisturí (EMB).

FIgURE 4. Cumulative germination curves for a) Maihuenia patagonica and b) Maihueniopsis darwinii var. hickenii, in control seeds (C) and seeds subjected to three pre-germinative treatments: cold moist stratification for 40 days (EHF), mechanical scarification with sand and sandpaper (EML), and mechanical scarification with a scalpel (EMB). 
TABLA II. Tiempo medio de germinación (media $\pm 1 \mathrm{DE}$ ) (TMG) y días transcurridos hasta el inicio de la germinación (IG) para Maihuenia patagonica y Maihueniopsis darwinii var. hickenii, en el control (C) y luego de la aplicación de tres tratamientos pre-germinativos: estratificación húmeda fría durante 40 días (EHF), escarificación mecánica con lija y arena (EML), y escarificación mecánica con bisturí (EMB). Para los casos en que las variables difirieron entre tratamientos, letras diferentes indican diferencias estadísticamente significativas dentro de una misma columna $(\mathrm{p}<0,05)$.

TABLE II. Mean germination time (mean \pm 1SD) and time until germination (IG) in control seeds (C) of Maihuenia patagonica and Maihueniopsis darwinii var. hickenii, and seeds subjected to three pre-germinative treatments: cold moist stratification for 40 days (EHF), mechanical scarification with sand and sandpaper (EML), and mechanical scarification with a scalpel (EMB). For cases in which variables differed between treatments, different letters indicate statistically significant differences within the same column $(\mathrm{p}<0.05)$.

\begin{tabular}{c|c|c|c|c}
\hline \multirow{2}{*}{} & \multicolumn{2}{|c|}{ Maihuenia patagonica } & \multicolumn{2}{c}{ Maihueniopsis darwinii var. hickenii } \\
\cline { 2 - 4 } & TMG & IG & TMG & IG \\
\hline C & $17,3 \pm 6,2 \mathrm{a}$ & $11,6 \pm 3,9 \mathrm{a}$ & $30,5 \pm 5,2$ & $20,0 \pm 2,9 \mathrm{ac}$ \\
$\mathrm{EHF}$ & $31,2 \pm 7,6 \mathrm{~b}$ & $18,8 \pm 5,5 \mathrm{~b}$ & $28,7 \pm 8,2$ & $11,4 \pm 6,0 \mathrm{~b}$ \\
$\mathrm{EML}$ & $24,0 \pm 12,3 \mathrm{ab}$ & $11,8 \pm 2,9 \mathrm{ab}$ & $34,8 \pm 3,3$ & $24,6 \pm 3,1 \mathrm{a}$ \\
$\mathrm{EMB}$ & $17,2 \pm 4,4 \mathrm{a}$ & $9,0 \pm 1,6 \mathrm{a}$ & $29,4 \pm 6,6$ & $17,8 \pm 4,1 \mathrm{c}$ \\
\hline
\end{tabular}

\section{DISCUSIÓN}

Las semillas de $M$. patagonica y $M$. darwinii presentaron elevada viabilidad, sin embargo, la germinación fue baja en comparación con otras especies emparentadas (Kwack \& Zimmer 1978, Flores et al. 2011). Con relación a los tratamientos pregerminativos aplicados, la estratificación y la escarificación con lija y arena no tuvieron efecto en ninguna de las especies, mientras que la escarificación con bisturí aumentó el porcentaje de germinación, pero solo en el caso de $M$. patagonica.

Los porcentajes de germinación obtenidos son relativamente bajos comparados con lo observado para otras especies de Maihuenia y Maihueniopsis. Por ejemplo, para Maihuenia poeppigii se han observado porcentajes de germinación superiores al 90\% (Kwack \& Zimmer 1978, Flores et al. 2011), y para Maihueniopsis camachoi (Espinosa) F.Ritter y M. ovata (Pfeiff.) F. Ritter 50 y 65\%, respectivamente, en condiciones de luz (Flores et al. 2011). Estos resultados pueden atribuirse a distintos factores, como el régimen de temperatura utilizado, la edad de las semillas, es decir, el tiempo transcurrido entre su colecta y el desarrollo del ensayo de germinación, y el hecho de que las mismas fueron extraídas de frutos húmedos. Si bien el régimen de temperaturas utilizado $\left(20 / 10^{\circ} \mathrm{C}\right)$ es representativo del área de estudio (ver por ejemplo Masini et al. 2012), las temperaturas empleadas son menores a la temperatura óptima reportada para otras especies de cactus, como Trichocereus terscheckii (Pfeiff.) Britton \& Rose $\left(15-30{ }^{\circ} \mathrm{C}\right)$ (Ortega-Baes \& Rojas-Aréchiga 2007), Gymnocalycium schickendantzii (F.A.C. Weber) Britton \& Rose $\left(30^{\circ} \mathrm{C}\right.$ ) (Méndez 2011), y distintas especies de Opuntia (20-30 ${ }^{\circ} \mathrm{C}$ ) (Reyes-Agüero et al. 2006). Por otro lado, un régimen de alternancia de temperaturas puede favorecer, disminuir o no afectar la germinación en comparación con una temperatura constante (Rojas-Aréchiga \& VázquezYanes 2000). Por ejemplo, para $M$. ovata se ha observado un $75 \%$ de germinación tanto a $25^{\circ} \mathrm{C}$ como a $30 / 20{ }^{\circ} \mathrm{C}$, con fotoperíodo de 8/16 h (Royal Botanic Gardens Kew 2008).

Para varias especies de cactus es necesario un período de maduración o envejecimiento luego de la cosecha para que ocurra germinación o ésta sea elevada (Rojas-Aréchiga \& Vázquez-Yanes 2000). De presentar dicho requerimiento, es posible que las semillas de $M$. patagonica y de $M$. darwinii no hayan tenido tiempo suficiente y/o no hayan sido sometidas a las condiciones adecuadas de temperatura para desarrollar dicha maduración, ya que fueron almacenadas durante 60 días en frío $\left(5^{\circ} \mathrm{C}\right)$ previo a la realización del ensayo. Sin embargo, semillas de $M$. patagonica almacenadas 11 meses a temperatura ambiente y luego dos meses en frío germinaron menos de un 10\% (Leuenberger 1997), presentando un resultado similar al observado en este trabajo. Existe la posibilidad de que el almacenamiento a baja temperatura genere cambios fisiológicos que modifiquen la respuesta germinativa en comparación con semillas recién recolectadas (Baskin \& Baskin 1998).

Las semillas de ambas especies fueron extraídas de frutos carnosos, los cuales pueden presentar sustancias inhibidoras de la germinación. La existencia de mecanismos hormonales que evitan la germinación de las semillas en el interior de los frutos ha sido observada en distintas especies (ver por ejemplo Kobayashi et al. 2010), y sustancias inhibidoras de la germinación han sido detectadas en el tegumento de semillas de cactáceas, las cuales requieren de lavado o imbibición para eliminar dichas sustancias y obtener elevadas tasas de germinación (Rojas-Aréchiga \& VázquezYanes 2000). Por ejemplo, semillas de Maihueniopsis atacamensis (Phil.) F. Ritter y M. conoidea (Backeb.) F. Ritter sometidas a un pretratamiento de imbibición durante 10 y 11 semanas, respectivamente, y luego a un tratamiento 
de escarificación germinaron alrededor de un 80\% (Royal Botanic Gardens Kew 2008). Alternativamente, puede ser necesaria la presencia de sustancias que estimulen la germinación, como en el caso de Denmoza rhodacantha (Salm-Dyck) Britton \& Rose, que requiere de la aplicación combinada de escarificación mecánica y una solución de calcio, condición observada en su hábitat natural en los sitios donde ocurre el reclutamiento de nuevas plantas (Méndez 2007). También se ha documentado la acción de hongos promotores de la germinación en especies del género Opuntia (Delgado-Sánchez et al. 2013).

La ausencia de diferencias entre el tratamiento de estratificación y el control en ambas especies indica la ausencia de un mecanismo de dormancia fisiológica que sea superado mediante dicho tratamiento $\left(5^{\circ} \mathrm{C}\right.$ durante 40 días). En el caso de M. patagonica, esto contrasta con lo observado para $M$. poeppigii, cuyas semillas estratificadas a $5{ }^{\circ} \mathrm{C}$ durante 40 días presentaron una germinación mayor al $60 \%$ (Kwack \& Zimmer 1978). Si bien Kwack \& Zimmer (1978) no incluyeron un control, otros trabajos observaron que semillas nuevas de $M$. poeppigii solamente germinan luego de la aplicación de este pre-tratamiento (Leuenberger 1997), a diferencia de las semillas de $M$. patagonica que germinaron un $8,7 \pm 4,2 \%$ en el control. Por otro lado, dado que la escarificación con arena y lija no difirió del control para ninguna de las especies, este no resulta un tratamiento adecuado para procesar lotes de semillas con fines de restauración.

La mayor germinación obtenida en la escarificación con bisturí para $M$. patagonica indica que el tegumento evita la germinación de las semillas en alguna medida. En el caso de $M$. darwinii, la ausencia de diferencias entre la escarificación con bisturí y el control podría atribuirse a que el tratamiento de escarificación solamente retiró parte del arilo que rodea a la semilla, sin afectar la integridad del tegumento que, a pesar de ser delgado y débil (Kiesling 1984), podría ser igualmente impermeable. De hecho, se obtuvieron indicios de esto durante la realización de la prueba de viabilidad de tetrazolio, ya que debió retirarse parte del tegumento y dejarse expuesto el embrión para que fuera posible la tinción del mismo. De esta manera, no solo el arilo sino también el tegumento constituyen una estructura de resistencia a la germinación en $M$. darwinii. Para ambas especies, la ocurrencia de germinación en el control descartaría la ocurrencia de dormancia física, ya que las semillas logran embeberse igualmente en ausencia de escarificación. Adicionalmente, debe mencionarse que las cactáceas pertenecen al grupo de Angiospermas con semillas de embrión periférico, caracterizado por la ausencia de mecanismos de dormancia o por la ocurrencia de dormancia fisiológica (Finch-Savage \& Leubner-Metzger 2006). Ya que la escarificación también estimula la germinación en semillas con dormancia fisiológica leve (Baskin \& Baskin
2004), los resultados observados para $M$. patagonica serían acordes con este tipo de mecanismo.

Los porcentajes de germinación no se correspondieron con el tiempo que tardan las semillas en germinar, ya que por ejemplo el tratamiento de escarificación con bisturí en M. patagonica presentó mayor porcentaje de germinación pero no un menor TMG o IG. Esto puede deberse a que la velocidad de germinación es controlada principalmente por la temperatura y la edad de las semillas (Rojas-Aréchiga \& Vázquez-Yanes 2000), que no diferían entre las semillas del control y los tratamientos de escarificación. Sin embargo, los resultados obtenidos en el tratamiento de estratificación son menos claros, ya que en el caso de M. patagonica la exposición al frío generó una respuesta más lenta por parte de las semillas, mientras que para $M$. darwinii dicha respuesta se vio acelerada, lo cual puede relacionarse con la distribución altitudinal de esta especie. En el caso de $M$. patagonica, tanto el tiempo transcurrido hasta el inicio de la germinación (18,8 $\pm 5,5$ días $)$ como el tiempo medio de germinación ( $31,2 \pm 7,6$ días) en el tratamiento de estratificación fue elevado en comparación a lo observado para M. poeppigii, que presentó en semillas recientemente colectadas valores de IG de entre 3-8 días y de TMG de entre 8-18 días, para semillas expuestas a temperaturas de estratificación de 5,10 y $15^{\circ} \mathrm{C}$ (Kwack \& Zimmer 1978).

En conclusión, los resultados obtenidos sugieren que tanto M. patagonica como M. darwinii presentan algún mecanismo de dormancia, ya que se obtuvieron bajos porcentajes de germinación aun cuando las semillas de ambas especies se encontraban viables. Se descarta la existencia de dormancia física debido a que hubo germinación (y por ende imbibición) en el control para ambas especies, y se propone la existencia de un mecanismo de dormancia fisiológica leve. Esta habría sido superada parcialmente por la escarificación mecánica con bisturí del tegumento en $M$. patagonica. Sin embargo, debería evaluarse la germinación de semillas sometidas a un período de maduración a temperatura ambiente para poder confirmar esta hipótesis. Alternativamente, los bajos porcentajes de germinación obtenidos también podrían atribuirse a la presencia de sustancias inhibidoras de la germinación que necesitan ser lavadas y a la utilización de un rango de temperaturas demasiado bajas.

El presente trabajo constituye un nuevo aporte al estudio de los cactus de Sudamérica y con forma de crecimiento no columnar, ambos grupos poco representados en la bibliografía de las cactáceas (Godínez-Álvarez et al. 2003). Provee información sobre la fisiología básica de $M$. patagonica y $M$. darwinii, que resulta útil para su propagación a partir de semillas y posterior utilización en trabajos de restauración de hábitats naturales degradados. Esta contribución es valiosa para la conservación in situ de las cactáceas estudiadas, dado el papel clave que desempeña este grupo en los ecosistemas áridos. 


\section{AGRADECIMIENTOS}

A Martín Wesley, Sergio Goitia, Alberto Galussi y Daniel Testoni por su colaboración en distintas etapas del trabajo. A Mariana Rojas Aréchiga y revisores anónimos por la revisión del manuscrito. PIP 11420100100258.

\section{BIBLIOGRAFÍA}

Anderson, E.F. 2001. The Cactus Family. Timber Press, Portland. $776 \mathrm{pp}$.

Bacilio, M., J.P. Hernández \& Y. Bashan. 2006. Restoration of giant cardon cacti in barren desert soil amended with common compost and inoculated with Azospirillum brasilense. Biology and Fertility of Soils 43(1): 112-119.

Baingridge, D. 2007. A Guide for Desert and Dryland Restoration: New hope for Arid Lands. Island Press, Washington. 391 pp.

Baskin, C.C. \& J.M. Baskin. 1998. Seeds: Ecology, Biogeography, and Evolution of Dormancy and Germination. Academic Press, San Diego. 666 pp.

BASKIn, J.M. \& C.C. BASKIN. 2004. A classification system for seed dormancy. Seed Science Research 14(1): 1-16.

Butterworth, C.A. \& R.S. Wallace. 2005. Molecular phylogenetics of the leafy cactus genus Pereskia (Cactaceae). Systematic Botany 30(4): 800-808.

Cabrera, A.L. 1971. Fitogeografía de la República Argentina. Boletín de la Sociedad Argentina de Botánica 14(1-2):142.

Cazón, A., M.L. de Viana \& J.C. Gianello. 2002. Comparación del efecto fitotóxico de aleloquímicos de Baccharis boliviensis (Asteraceae) en la germinación de Trichocereus pasacana (Cactaceae). Ecología Austral 12(1): 73-78.

CITES. 2013. Convention On International Trade In Endangered Species Of Wild Fauna and Flora - Appendices I, II and III. URL: http://www.cites.org/eng/app/2013/EAppendices-2013-06-12.pdf Viewed: September 25, 2013.

Commander, L.E., D.J. Merritt, D.P. Rokich \& K.W. Dixon. 2009. Seed biology of Australian arid zone species : Germination of 18 species used for rehabilitation. Journal of Arid Environments 73(6-7): 617-625.

Delgado-Sánchez, P., M.A. Ortega-Amaro, J.F. Jiménez-Bremont \& J. Flores. 2011. Are fungi important for breaking seed dormancy in desert species? Experimental evidence in Opuntia streptacantha (Cactaceae). Plant Biology 13(1): 154-159.

Delgado-Sánchez, P., J.F. Jiménez-Bremont, M.L. GuerreroGonZÁlez \& J. Flores. 2013. Effect of fungi and light on seed germination of three Opuntia species from semiarid lands of central Mexico. Journal of Plant Research 126(5): 643-649.

Demaio, P., R. Kiesling, M. Lowry, E. Méndez, P. Saldivia, L. FAÚNDEZ \& H.E. Walter. 2013. Maihueniopsis darwinii. URL: $\quad$ http://www.iucnredlist.org/details/151977/0 Viewed: September 26, 2013.

Departamento de Geografía UNCo. 1982. Atlas de la Provincia del Neuquén. 185 pp.

Finch-Savage, W.E. \& G. Leubner-Metzger. 2006. Seed dormancy and the control of germination. The New phytologist 171(3): 501-523.

Fiori, S.M. \& S.M. ZALBA. 2003. Potential impacts of petroleum exploration and exploitation on biodiversity in a Patagonian Nature Reserve, Argentina. Biodiversity and Conservation 12(6): 1261-1270.

Flores, J., A. Arredondo \& E. Jurado. 2005. Comparative seed germination in species of Turbinicarpus: an endangered cacti genus. Natural Areas Journal 25(2): 183-187.

Flores, J., E. Jurado, L. Chapa-Vargas, A. Ceroni-Stuva, P. Dávila-Aranda, G. Galíndez, D. Gurvich, P. LeÓn-Lobos, C. Ordoñes, P. Ortega-Baes, N. Ramírez-Bullon, A. Sandoval, C.E. Seal, T. Ullian \& H.W. Pritchard. 2011. Seeds photoblastism and its relationship with some plant traits in 136 cacti taxa. Environmental and Experimental Botany 71(1): 79-88.

Godínez-Álvarez, H., T. Valverde \& P. Ortega-Baes. 2003. Demographic trends in the Cactaceae. The Botanical Review 69(2): 173-203.

Gosling, P.G. 2003. Viability Testing. In: R.D. Smith, J.B. Dickie, S.H. Linington, H.W. Pritchard \& R.J. Probert (eds.), Seed Conservation: turning science into practice, pp. 445-481. The Royal Botanic Gardens. Kew, Great Britain.

Guerrant JR., E.O. \& A. Raven. 2003. Supporting In situ Conservation: The Berry Botanic Garden, an ex situ regional resource in an integrated conservation community. In: R.D. Smith, J.B. Dickie, S.H. Linington, H.W. Pritchard \& R.J. Probert (eds.), Seed Conservation: turning science into practice. pp. 879-896. The Royal Botanic Gardens, Kew, Great Britain.

Gurvich, D.E., G. Funes, M.A. Giorgis \& P. Demaio. 2008. Germination characteristics of four argentinean endemic Gymnocalycium (Cactaceae) species with different flowering phenologies. Natural Areas Journal 28(2): 104108.

Hartmann, H.T. \& D.E. Kester. 1980. Propagacion de plantas: principios y prácticas. Compañía Editorial Continental S.A., México. 814 pp.

Kiesling, R. 1975. Los géneros de Cactaceae de Argentina. Boletín de la Sociedad Argentina de Botánica 16(3): 197-227.

Kiesling, R. 1984. Estudios de Cactaceae de Argentina: Maihueniopsis, Tephrocactus y géneros afines (Opuntioideae). Darwiniana 25(1-4): 171-215.

Kiesling, R. 1988. Cactaceae. En: M.N. Correa (ed.), Flora Patagónica - Parte V: Dicotiledóneas Dialipetalas (Oxalidaceae a Cornaceae), pp. 218-243. Colección Científica del INTA Tomo VIII, Buenos Aires, Argentina.

Kiesling, R., L. Faúndez, P. Saldivia \& H.E. Walter. 2013. Maihuenia patagonica. URL: http://www.iucnredlist.org/ details/summary/152787/0 Viewed: September 26, 2013.

Kobayashi, Y., K. Nabeta \& H. Matsuura. 2010. Chemical inhibitors of viviparous germination in the fruit of watermelon. Plant \& Cell Physiology 51(9):1594-1598.

Kwack, B.H. \& K. Zimmer. 1978. Germination of aged seeds of Maihuenia poeppigii. Gartenbauwissenschaft 43(4): 188191.

Las Peñas, M.L., J.D. Urdampilleta, B. López-Carro, F. Santiñaque, R. Kiesling \& G. Bernardello. 2014. Classical and molecular cytogenetics and DNA content in Maihuenia and Pereskia (Cactaceae). Plant Systematics 
and Evolution 300(3): 549-558.

Leuenberger, B.E. 1997. Maihuenia - monograph of a Patagonian genus of Cactaceae. Botanische Jahrbücher für Systematik 119(1): 1-92.

Martínez Carretero, E. 2004. La Provincia fitogeográfica de la Payunia. Boletín de la Sociedad Argentina de Botánica 39(3-4): 195-226.

Martínez Pérez, G., A. Orozco-Segovia \& C. Martorell. 2006. Efectividad de algunos tratamientos pre-germinativos para ocho especies leñosas de la Mixteca Alta oaxaqueña con características relevantes para la restauración. Boletín de la Sociedad Botánica de Mexico 79: 9-20.

Masini, A.C.A., A.E. Rovere \& D.R. Perez. 2012. Requerimientos pregerminativos de dos especies leñosas: Anarthrophyllum capitatum Sorarú y Anarthrophyllum elegans (Gillies ex Hook. \& Arn.) F. Philippi. Quebracho 20(1,2): 85-96.

Mauseth, J.D. 1999. Anatomical Adaptations to xeric conditions in Maihuenia (Cactaceae), a relictual, leaf-bearing cactus. Journal of Plant Research 112(3):307-315.

Méndez, E. 2007. Germination of Denmoza rhodacantha (SalmDyck) Britton \& Rose (Cactaceae). Journal of Arid Environments 68(4): 678-682.

MÉnDEZ, E. 2011. Efecto de la temperatura, escarificación y concentraciones de calcio en la germinación de Gymnocalycium schickendantzii (F.A.C. Weber) Britton \& Rose (Cactaceae ). Revista de la Facultad de Ciencias Agrarias UNCUYO 43(2):103-109.

Méndez, E., J. Guevara \& O. Estevez. 2004. Distribution of cacti in Larrea spp. shrublands in Mendoza, Argentina. Journal of Arid Environments 58(4): 451-462.

Méndez, E. \& S.B. Pérez GonzÁlez. 2008. Germinación de Echinopsis leucantha (Cactaceae). I-Efectos de temperatura y concentraciones de calcio. Revista de la Facultad de Ciencias Agrarias UNCUYO 40(2): 91-96.

Oldfield, S. 1997. Cactus and Succulent Plants - Status Survey and Conservation Action Plan. IUCN, Gland, Switzerland and Cambridge, UK. 312 pp.

Ortega-Baes, P. \& H. Godínez-Álvarez. 2006. Global Diversity and Conservation Priorities in the Cactaceae. Biodiversity and Conservation 15(3): 817-827.

Ortega-Baes, P. \& M. Rojas-Aréchiga. 2007. Seed germination of Trichocereus terscheckii (Cactaceae): Light, temperature and gibberellic acid effects. Journal of Arid Environments 69(1): 169-176.

Ortega-Baes, P., M. Aparicio \& G. Galíndez. 2010a. Vivipary in the cactus family: An evaluation of 25 species from northwestern Argentina. Journal of Arid Environments 74(10): 1359-1361.

Ortega-Baes, P., M. Aparicio-González, G. Galíndez, P. del Fueyo, S. Sühring \& M. Rojas-Aréchiga. 2010b. Are cactus growth forms related to germination responses to light? A test using Echinopsis species. Acta Oecologica 36(3): 339-342.

Ortega-Baes, P., S. Sühring, J. Sajama, E. Sotola, M. AlonsoPedano, S. Bravo \& H. Godínez-Álvarez. 2010c. Diversity and Conservation in the Cactus Family. In: K.G. Ramawat (ed.), Desert Plants: Biology and Biotechnology, pp. 157-
173. Springer Berlin Heidelberg, Berlin.

Paruelo, J.M., A. Beltrán, E. Jobbágy, O.E. Sala \& R.A. Golluscio. 1998. The climate of Patagonia: general patterns and controls on biotic processes. Ecología Austral 8(2): 85-101.

PlanEAr. 2008. Maihueniopsis darwinii (Hensl.) F. Ritter. URL: http://www.lista-planear.org/index. php?item $=$ especie\&accion $=$ ver_ficha\&id $=28762$ Viewed: September 26, 2013.

Reyes-Agüero, J.A., J.R. Aguirre \& A. Valiente-Banuet. 2006. Reproductive biology of Opuntia: A review. Journal of Arid Environments 64(4): 549-585.

Rojas-Aréchiga, M. \& C. VÁzquez-Yanes. 2000. Cactus seed germination: a review. Journal of Arid Environments 44(1): 85-104.

Rojas-Aréchiga, M., M.C. Mandujano \& J.K. Golubov. 2013. Seed size and photoblastism in species belonging to tribe Cacteae (Cactaceae). Journal of Plant Research 126(3): 373-86.

Royal Botanic Gardens Kew. 2008. Seed Information Database (SID). Version 7.1. URL: http://data.kew.org/sid/ Viewed: October 7, 2013.

Sánchez, A.M., F.M. Azcarate, L. Arqueros \& B. Peco. 2002. Volumen y dimensiones como predictores del peso de semilla de especies herbáceas del centro de la Península Ibérica. Anales Jardín Botánico de Madrid 59(2): 249-262.

Siegel, S. \& N.J. Castellan. 1995. Estadística No Parametrica: Aplicada a las ciencias de la conducta. 4a ed., Trillas, México. 437 pp.

Stiling, P., A. Rossi \& D. Gordon. 2000. The difficulties of single factor thinking in restoration: replanting a rare cactus in the Florida Keys. Biological Conservation 94(3): 327-333.

Terry, J., R.J. Probert \& S.H. Linington. 2003. Processing and Maintenance of the Millennium Seed Bank Collections. In: R.D. Smith, J.B. Dickie, S.H. Linington, H.W. Pritchard \& R.J. Probert (eds.), Seed Conservation: turning science into practice. pp. 307-325. The Royal Botanic Gardens, Kew, Great Britain.

Way, M.J. 2003. Collecting Seed from Non-domesticated Plants for Long-Term Conservation. In: R.D. Smith, J.B. Dickie, S.H. Linington, H.W. Pritchard \& R.J. Probert (eds.), Seed Conservation: turning science into practice. pp. 163-201. The Royal Botanic Gardens, Kew, Great Britain.

Woods, S.R., J.S. FeHMi \& D.M. BACKER. 2012. An assessment of revegetation treatments following removal of invasive Pennisetum ciliare (buffelgrass). Journal of Arid Environments 87: 168-175.

Yang, X., H.W. Pritchard \& H. Nolasco. 2003. Effects of Temperature on Seed Germination in Six Species of Mexican Cactaceae. In: R.D. Smith, J.B. Dickie, S.H. Linington, H.W. Pritchard \& R.J. Probert (eds.), Seed Conservation: turning science into practice, pp. 575-588. The Royal Botanic Gardens. Kew, Great Britain.

Zuloaga, F.O., O. Morrone \& M.J. Belgrano. 2008. Catálogo de las Plantas Vasculares del Cono Sur (Argentina, Sur de Brasil, Chile, Paraguay y Uruguay). Missouri Botanical Garden Press, St. Louis. 3348 pp.

Recibido: 21.01 .14

Aceptado: 23.04.14 